\title{
CXC Chemokine Receptor 2 Antagonist AZD5069
}

National Cancer Institute

\section{Source}

National Cancer Institute. CXC Chemokine Receptor 2 Antagonist AZD5069. NCI

Thesaurus. Code C123383.

An orally bioavailable, selective and reversible antagonist of CXC chemokine receptor 2 (CXCR2), with potential anti-inflammatory and antineoplastic activities. Upon administration, CXC chemokine receptor 2 antagonist AZD5069 directly binds to CXCR2 and inhibits its activation. This inhibits CXCR2-mediated signaling and may inhibit tumor cell proliferation in CXCR2-overexpressing tumor cells. In addition, AZD5069 reduces both neutrophil recruitment and migration from the systemic circulation into sites of inflammation, including the lung mucosa; it may also prevent neutrophil migration from the bone marrow. This results in the reduction of inflammation, mucus production, and neutrophil proteinase-mediated tissue destruction in the lung. CXCR2, a G proteincoupled receptor protein also known as IL-8 receptor B (IL-8RB), is upregulated in a variety of tumor cell types and plays a key role in tumor cell proliferation and progression; it is known to be elevated in several inflammatory diseases, such as chronic obstructive pulmonary disease (COPD), asthma and fibrotic pulmonary disorders. 